\title{
Memorial address for Togo Horiuchi (1924-2012)
}

\author{
Yoshikatsu Saiki
}

Published online: 10 December 2013

(C) The Japanese Association for Thoracic Surgery 2013

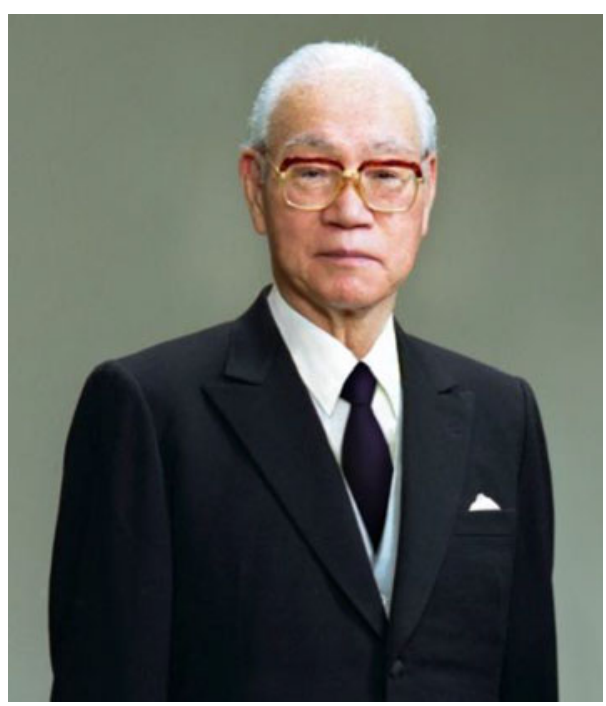

The late Dr. Togo Horiuchi significantly contributed to the establishment and development of the Tohoku University Cardiovascular Surgery Department. Although the history of cardiovascular surgery in the Tohoku University School of Medicine began in the latter half of 1950s, full-fledged operation began after the Cardiothoracic Surgery Department was established in 1971 with the cooperation of the First and Second Departments of Surgery.

After graduating from the Tohoku University School of Medicine, Dr. Horiuchi joined the Second Department of Surgery at Tohoku University and was a member of

Y. Saiki $(\bowtie)$

1-1 Seiryocho, Aobaku, Sendai, Miyagi, Japan

e-mail: yoshisaiki@med.tohoku.ac.jp the heart and vascular surgery research group. After completing his dissertation entitled "Experimental study of cardiac resuscitation", he took up a position at the Koshirakawa Shiseido Hospital in Yamagata Prefecture in 1954. In spite of the insufficient facilities and shortages of human resources there, he achieved a brilliant level of performance including 39 consecutive successes in surgery with no cases of death, which was an astounding record at that time. It was a tremendous achievement specifically because it was accomplished at one of the local private hospitals as opposed to a university hospital.

After moving back to the Second Department of Surgery at the Tohoku University School of Medicine in 1959, he published new methods of surgery such as "Bypass deep hypothermia" and "Tracheary graft with autologous pericardial valve". In 1975, he was elected as the first professor in the Cardiothoracic Surgery Department.

Dr. Horiuchi made enormous efforts in the development of surgical treatment of congenital cardiac diseases and rendered distinguished services, one of which was his manuscript entitled "Operation for the radical correction for deficiency of the cardiac chamber" which was regarded with high esteem as one of the 26 important clinical advances in cardiovascular and pulmonary medicine and surgery during the 30 years since the end of world war II by the U.S. National Institutes of Health. He also was involved in the study of the artificial heart and mechanical circulatory assist device early on, as he reported the first case of implantation of a ventricular assist device and its successful removal after the recovery of cardiac function.

His concept for surgical operation was a perfect balance between "technical excellence" and "tenderness"-in other words, "skill with cordiality," which had something 
in common with the teaching of Ryokan, a Zen master of the Edo era: "artistic quality full of humanity and life based on religious ideas".
With all his brilliant scientific achievements, Dr. Horiuchi will continue to deserve the admiration of every cardiovascular surgeon in the world. May his soul rest in peace. 